06

\title{
Нелинейная рефракция диэлектрических монокристаллов при возбуждении ультракотроткими лазерными импульсами ближнего инфракрасного диапазона
}

\author{
(C) А.И. Водчиц ${ }^{1}$, В.С. Горелик ${ }^{2,3}$, В.А. Орлович ${ }^{1}$, П.А. Апанасевич ${ }^{1}$ \\ ${ }^{1}$ Институт фризики им. Б.И. Степанова НАН Беларуси, \\ 220072 Минск, Беларусь \\ ${ }^{2}$ Физический институт им. П.Н. Лебедева РАН, \\ 119991 Москва, Россия \\ ${ }^{3}$ МГТУ им. Н.Э. Баумана, \\ 105005 Москва, Россия \\ e-mail: gorelik@sci.lebedev.ru
}

Поступила в редакцию 03.06.2019 г.

В окончательной редакции 03.06.2019 г.

Принята к публикации 11.06.2019 г.

Методом однопучкового Z-сканирования исследована нелинейная рефракция в ряде кристаллов (KGW, $\left.\mathrm{KGW}: \mathrm{Eu}^{3+}, \mathrm{Ba}\left(\mathrm{NO}_{3}\right)_{2}, \mathrm{BaWO}_{4}, \mathrm{PbMoO}_{4}, \mathrm{CdWO}_{4}\right)$, перспективных для возбуждения многочастотного вынужденного комбинационного рассеяния света. Для возбуждения нелинейно-оптических процессов использовались пикосекундные лазерные импульсы лазера $\mathrm{YAG}: \mathrm{Nd}^{3+}$, генерирующего излучение на длине волны $1064 \mathrm{~nm}$. Выполнено сопоставление величин $n_{2}$ в зависимости от типа кристалла, от ориентации оптической оси и от концентрации примесей.

Ключевые слова: нелинейная рефракция, нелинейное поглощение, вынужденное комбинационное рассеяние, лазер, Z-сканирование, пикосекундный импульс, диафрагма, детектор.

DOI: $10.21883 /$ OS.2019.10.48359.173-19

\section{Введение}

К настоящему времени разработана технология выращивания совершенных монокристаллов, перспективных для нелинейно-оптических приложений. Особый класс нелинейно-оптических кристаллов формируется на основе диэлектрических структур, перспективных для возбуждения процессов вынужденного комбинационного рассеяния (ВКР) [1-7]. Среди такого типа кристаллов большой практический интерес представляют следующие: $\mathrm{KGW}, \mathrm{KGW}$, легированный ионами европия $\left(\mathrm{KGW}: \mathrm{Eu}^{3+}\right)$, нитрат бария $\left(\mathrm{Ba}\left(\mathrm{NO}_{3}\right)_{2}\right)$, вольфрамат бария $\left(\mathrm{BaWO}_{4}\right)$, вольфрамат кадмия $\left(\mathrm{CdWO}_{4}\right)$ и молибдат свинца $\left(\mathrm{PbMoO}_{4}\right)$. Генерационные параметры нелинейно-оптических и лазерных устройств, создаваемых на основе этих кристаллов, тесно связаны с их нелинейно-оптическими свойствами. К таким свойствам, в частности, относятся двухфотонно-возбуждаемая фотолюминесценция $[7,8]$, двухфотонное поглощение $[9,10]$, нелинейная рефракция [11-14], генерация высших оптических гармоник [15-17] и др. Нелинейная рефракция и нелинейное поглощение света приводят к искажению поперечного распределения поля в лазерном пучке, к изменению расходимости пучка на выходе из образца, к проявлению эффектов самофокусировки и др. Нелинейное поглощение интенсивного лазерного излучения в конденсированной диэлектрической среде обусловлено главным образом процессами двухфотонного поглоще- ния, в наибольшей степени проявляющимися при попадании удвоенной частоты лазерного излучения в область частот электронных переходов среды, расположенных в фиолетовой или ультрафиолетовой областях спектра. Информация о величине нелинейного показателя преломления и нелинейного показателя поглощения имеет большое значение при проектировании нелинейнооптических и лазерных устройств с использованием обсуждаемых конденсированных диэлектрических сред.

В настоящей работе приводятся результаты экспериментальных исследований процессов нелинейной рефракции и двухфотонного поглощения в упомянутых выше кристаллах при возбуждении этих процессов интенсивным лазерным излучением ближнего инфракрасного (ИК) диапазона. Для измерений нелинейного показателя преломления кристаллов в наших исследованиях применялся известный однопучковый метод $Z$-сканирования [11-14].

\section{Методика эксперимента}

В экспериментах по исследованию нелинейной рефракции и двухфотонного поглощения использовался пикосекундный лазер на алюмоиттриевом гранате $\left(\right.$ YAG : $\mathrm{Nd}^{3+}$ ) на основе прибора LS-2151, LOTIS TII (Беларусь). Лазер генерировал импульсы длительностью 80 ps на длине волны $1064 \mathrm{~nm}$ и 60 ps на длине волны второй оптической гармоники $(532 \mathrm{~nm})$. Частота 


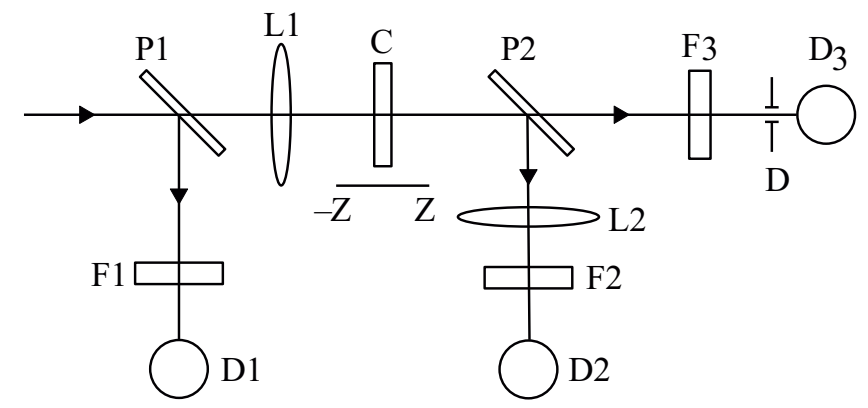

Pис. 1. Оптическая схема однопучкового $Z$-сканирования: P1,P2 - плоскопараллельные кварцевые пластинки; F1, F2, F3 - фильтры; L1, L2 - линзы; C - исследуемый кристалл; D - диафрагма; D1, D2, D3 - фотодетекторы.

повторения импульсов составляла $20 \mathrm{~Hz}$. Оптическая схема однопучкового $Z$-сканирования представлена на рис. 1.

Лазерный пучок излучения, имеющего горизонтальную поляризацию, фокусировался в плоскопараллельные пластинки диэлектрических кристаллов с помощью сферической линзы с фокусным расстоянием $35 \mathrm{~cm}$. В области фокальной перетяжки интенсивность возбуждающего лазерного излучения составляла $10^{10}-10^{11} \mathrm{~W} / \mathrm{cm}^{2}$. При перемещении линзы L1 в Z-направлении (рис. 1) в исследуемых образцах в области фокальной перетяжки пучка образец испытывал воздействие лазерного излучения изменяющейся интенсивности. После образца использовались два канала регистрации: канал с диафрагмой D и канал без диафрагмирования пучка (рис. 1). При этом проводились исследования процессов нелинейной рефракции при использовании диафрагмы $\mathrm{D}$, a также двухфотонного поглощения (при отсутствии такой диафрагмы). Нелинейная рефракция приводит к изменению интенсивности излучения, прошедшего через диафрагму D. B канале без диафрагмирования изменение сигнала может быть обусловлено главным образом нелинейным поглощением. В канале измерения нелинейной рефракции устанавливалась диафрагма диаметром $2 \mathrm{~mm}$ на расстоянии $138 \mathrm{~cm}$ относительно центра фокальной перетяжки пучка. Кристаллы перемещались вдоль фокальной перетяжки в автоматизированном режиме с помощью шагового двигателя, управляемого от компьютера. Шаг перемещения составлял $0.5 \mathrm{~mm}$. Усреднение сигналов производилось по 100 лазерным вспышкам в каждой точке. Для сбора излучения от всего пучка в канале без диафрагмы устанавливалась дополнительная фокусирующая линза. Сигналы от детекторов D3 и D2 нормировались на сигнал „накачки“ - детектор D1. Полученные экспериментальные зависимости сигналов от положения $Z$ кристалла относительно центра перетяжки $(Z=0)$ моделировались с использованием теоретической модели, изложенной в $[12,13]$.

Толщина плоскопараллельных монокристаллических пластин составляла 1-4 mm. Кристаллы имели определенные срезы. Исследовались три кристалла $\mathrm{KGW}: \mathrm{Eu}^{3+}$ с концентрациями ионов $\mathrm{Eu}^{3+} 2,10$ и 25 at.\%. В качестве эталонного образца применялась тонкая пластинка плавленого кварца.

\section{Результаты и их обсуждение}

Нелинейный показатель преломления определяется из соотношения для нелинейного изменения фазы поля излучения $\Delta \Phi_{n r}[12,13]$ :

$$
\Delta \Phi_{n r}=(2 \pi / \lambda) l_{\mathrm{ef}} n_{2} I_{\omega}
$$

где $\lambda-$ длина волны лазерного излучения, $l_{\mathrm{ef}}=$ $=\left[1-\exp \left(-\alpha_{0} l\right)\right] / \alpha_{0}-$ эффективная длина кристалла, $l$ - физическая длина кристалла, $\alpha_{0}$ - линейный коэффициент поглощения кристалла, $I_{\omega}-$ интенсивность лазерного излучения в фокальной перетяжке пучка, $n_{2}$ - нелинейный показатель преломления.

Нелинейный коэффициент поглощения $(\beta)$ определяется с использованием следующего соотношения для нелинейного изменения фазы $\Delta \Phi_{n a}$ в этом случае [12-14]:

$$
2 \Delta \Phi_{n a}=\ln \left(1+l_{\mathrm{ef}} \beta I_{\omega}\right) .
$$

Для определения нелинейных показателей преломления и нелинейного коэффициента поглощения регистрируемые экспериментальные кривые Z-сканирования моделировались теоретическими зависимостями (1), (2) с учетом предположений, изложенных в [12-14]. Основные предположения состояли в следующем: возбуждающий лазерный пучок имеет гауссово пространственное распределение интенсивности излучения, эффективная длина исследуемых кристаллов существенно меньше длины фокальной перетяжки пучка, временная форма лазерного импульса является гауссовой. Данные предположения выполняются в условиях выполненного эксперимента. При этом мощность пикосекундного импульса $P$ вычисляется по формуле

$$
P=0.94\left(\frac{W}{\Delta t_{p}}\right),
$$

где $W-$ энергия импульса, $\Delta t_{p}-$ его временная длительность. Интенсивность лазерного излучения в пучке $I$ в фокальной перетяжке определяется с использованием следующего соотношения:

$$
I=\frac{P}{\pi\left(\frac{\omega_{0}^{2}}{2}\right)},
$$

где $\omega_{0}-$ радиус перетяжки гауссова пучка в точке $z=0$.

Отметим также, что измеряемые экспериментальные зависимости для нелинейной рефракции соответствуют средним значениям нелинейного показателя преломления. Его пиковое значение в $\sqrt{2}$ раз больше. В настоящей работе приводятся пиковые значения нелинейного показателя преломления. 
Согласно нашим измерениям, нелинейный показатель преломления плавленого кварца на длине волны $1064 \mathrm{~nm}$ составляет $n_{2}=2 \cdot 10^{-16} \mathrm{~cm}^{2} / \mathrm{W}$. Эта величина находится в хорошем согласии с данными других исследователей и предыдущими измерениями [12-14]. Исследования показали, что во всех исследованных кристаллах величина коэффициента нелинейного поглощения при возбуждении лазером с длиной волны $1064 \mathrm{~nm}$ очень мала и находится в пределах ошибки измерений. Такой результат обусловлен тем, что край полосы фундаментального электронного поглощения в исследованных материалах находился в коротковолновой области спектра. При этом двухфотонные переходы $(\lambda=532 \mathrm{~nm})$ попадают в область прозрачности анализируемых диэлектрических сред. В связи с этим процессы двухфотонновозбуждаемой люминесценции были сильно подавлены, и потери энергии лазерного пучка за счет перехода в тепло в обсуждаемых диэлектрических средах практически отсутствовали.

На рис. 2 представлена полученная экспериментальная зависимость для кристалла $\mathrm{KGW}$, зарегистрированная при Z-сканировании. Энергия импульсов накачки при этом составляла $54 \mu \mathrm{J}$. Ось „,“ кристалла была перпендикулярна вектору поляризации лазерного излучения. На представленном рисунке приведено нормированное пропускание сигнала через диафрагму в зависимости от координаты $Z$ (от центра фокальной перетяжки: $Z=0)$. Измеренный нелинейный показатель преломления оказался равным: $n_{2}=14 \cdot 10^{-16} \mathrm{~cm}^{2} / \mathrm{W}$. Это значение меньше по сравнению со значением на длине волны $790 \mathrm{~nm}$ [13], что обусловлено дисперсией $n_{2}$.

Поведение нелинейной рефракции (величины $n_{2}$ ) в кристаллах $\mathrm{KGW}: \mathrm{Eu}^{3+}$ оказалось более сложным: показатель нелинейной рефракции $n_{2}$ зависел от концентрации ионов европия и от ориентации оси „, $c^{\text {“ }}$ относительно поляризации лазерного излучения. Ориентационная зависимость $n_{2}$ становилась более выраженной при вы-

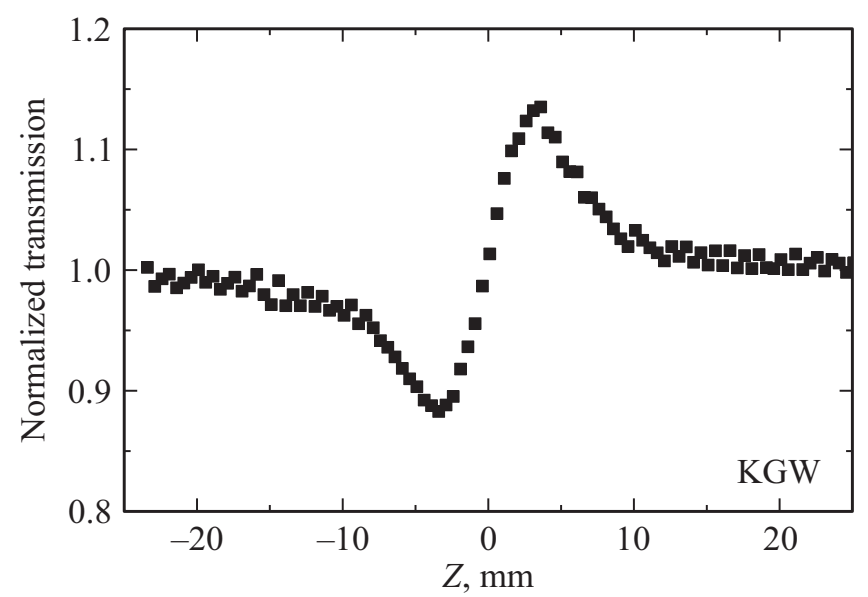

Рис. 2. Экспериментальная кривая $Z$-сканирования для кристалла KGW (ось „с“ кристалла перпендикулярна вектору поляризации лазерного излучения).

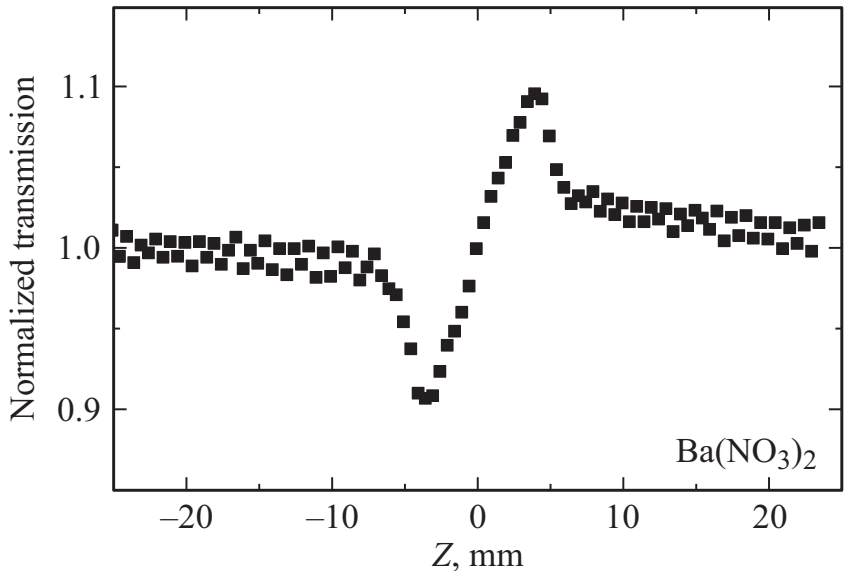

Рис. 3. Экспериментальная кривая Z-сканирования для кристалла нитрата бария.

сокой концентрации ионов. При этом увеличивалась разность между значениями $n_{2}$ при различных ориентациях оптической оси „с“ относительно вектора поляризации лазерного излучения. Значение $n_{2}$ было наименьшим в том случае, когда ось „с“ была параллельна вектору поляризации лазерного излучения и уменьшалось при более высоких концентрациях европия. Для другой ориентации оси „с“ нелинейная рефракция значительно увеличивалась. Такие закономерности свидетельствуют об анизотропии величины $n_{2}$ в одноосных кристаллах, а также о концентрационной зависимости этой величины от ионов $\mathrm{Eu}^{3+}$.

Нелинейная рефракция кристалла нитрата бария не зависела от ориентации кристалла относительно поляризации лазерного излучения, что связано с кубической симметрией соответствующей кристаллической структуры. Экспериментальные данные по $Z$-сканированию для нитрата бария иллюстрируются рис. 3. Энергия импульсов излучения при этом составляла $71 \mu \mathrm{J}$. Определяемое методом подгонки значение $n_{2}$ составило $6 \cdot 10^{-16} \mathrm{~cm}^{2} / \mathrm{W}$. Для вольфрамата бария нелинейный показатель преломления оказался равным $n_{2}=7 \cdot 10^{-16} \mathrm{~cm}^{2} / \mathrm{W}$. В вольфрамате кадмия наблюдалась существенно более сильная нелинейная рефракция. Для этого кристалла при параллельной ориентации оси „,“ и вектора поляризации излучения $n_{2}=14 \cdot 10^{-16} \mathrm{~cm}^{2} / \mathrm{W}$; при перпендикулярной ориентации $-n_{2}=17 \cdot 10^{-16} \mathrm{~cm}^{2} / \mathrm{W}$.

Сильная нелинейная рефракция наблюдалась в молибдате свинца. В $\mathrm{PbMoO}_{4}(a$-срез $) n_{2}=25 \cdot 10^{-16} \mathrm{~cm}^{2} / \mathrm{W}$ (при этом ось $a$ была параллельна вектору лазерной поляризации) и $n_{2}=28 \cdot 10^{-16} \mathrm{~cm}^{2} / \mathrm{W}$ для перпендикулярной ориентации. Для $\mathrm{PbMoO}_{4}$ (c-срез) было получено значение $n_{2}=30 \cdot 10^{-16} \mathrm{~cm}^{2} / \mathrm{W}$ для параллельной ориентации и $n_{2}=35 \cdot 10^{-16} \mathrm{~cm}^{2} / \mathrm{W}$ для перпендикулярной.

Полученные данные о величинах $n_{2}$ для всех исследованных кристаллов иллюстрируются таблицей. В этой таблице приведены значения $n_{2}$ для длины волны воз- 
Значения $n_{2}$ для исследованных кристаллов при использовании в качестве возбуждающего излучения ультракоротких интенсивных импульсов лазерного излучения с длиной волны $\lambda=1064 \mathrm{~nm}$

\begin{tabular}{c|l|c|c}
\hline № & \multicolumn{1}{|c|}{ Тип кристалла } & $\begin{array}{c}(g) n_{2} \cdot 10^{-16} \\
\mathrm{~cm}^{2} / \mathrm{W}\end{array}$ & $\begin{array}{c}(v) n_{2} \cdot 10^{-16} \\
\mathrm{~cm}^{2} / \mathrm{W}\end{array}$ \\
\hline 1 & $\mathrm{KGW}$ & 13 & 14 \\
2 & $\mathrm{KGW}: \mathrm{Eu}^{3+}, 2$ at.\% & 16 & 18 \\
3 & $\mathrm{KGW}: \mathrm{Eu}^{3+}, 10$ at.\% & 13 & 16 \\
4 & $\mathrm{KGW}: \mathrm{Eu}^{3+}, 25$ at.\% & 12 & 20 \\
5 & $\left.\mathrm{Ba} \mathrm{NO}_{3}\right)_{2}$ & 6 & 6 \\
6 & $\mathrm{BaWO}_{4}$ & 7 & 7 \\
7 & $\mathrm{CdWO}_{4}$ & 14 & 17 \\
8 & $\mathrm{PbMoO}_{4}(a$-сре3 $)$ & 25 & 28 \\
9 & $\mathrm{PbMoO}_{4}(c$-сре3) & 30 & 35
\end{tabular}

буждающего излучения $1064 \mathrm{~nm}$ для двух ориентаций оптических осей кристаллов относительно вектора поляризации лазерного излучения $(g-$ параллельная и $v$ - перпендикулярная ориентации).

\section{Заключение}

Таким образом, методом однопучкового Z-сканирования построены графики зависимости нормированной интенсивности направленного вторичного излучения от координаты $Z$, состоящие из областей ослабления и усиления (рис. 2 и 3) интенсивности излучения, регистрируемого детектором D3 (рис. 1). Получены количественные данные о нелинейной рефракции (о величинах $n_{2}$ ) для ряда ориентированных пластинок монокристаллов: $\mathrm{KGW}, \mathrm{KGW}: \mathrm{Eu}^{3+}$, $\mathrm{Ba}\left(\mathrm{NO}_{3}\right)_{2}, \mathrm{BaWO}_{4}, \mathrm{PbMoO}_{4}, \mathrm{CdWO}_{4}$. Исследованные кристаллы используются для создания многочастотных ВКР-лазеров, генерирующих стоксовы и антистоксовы спутники в ИК и видимом спектральных диапазонах. Исследования выполнены с использованием в качестве возбуждающего излучения ультракоротких импульсов лазера YAG : $\mathrm{Nd}^{3+}$ ближнего инфракрасного диапазона $(\lambda=1064 \mathrm{~nm})$, характеризующегося большой пиковой интенсивностью излучения и высокой плотностью мощности при фокусировке возбуждающего излучения в объеме образцов. Установлено, что при используемых режимах возбуждения двухфотонное поглощение в исследованных образцах практически не наблюдалось, что обусловлено прозрачностью этих кристаллов в области двухфотонных переходов $(\lambda=532 \mathrm{~nm})$ и низкой эффективностью нелинейно-возбуждаемой люминесценции. В связи с отсутствием двухфотонного поглощения главным механизмом для реализации нелинейной рефракции в данном случае остаются стоксовы и антистоксовы процессы ВКР, приводящие к квантовой интерференции, т.е. к фотон-бифотонной конверсии с образованием связанных состояний двух фотонов с антипараллельными направлениями спинов (векторов напряженности электрического поля). В результате образования связанных состояний двух фотонов [15-17] со скалярной поляризацией („темных фотонов“ „дарктонов“") и малой расходимостью соответствующего излучения происходит ослабление интенсивности возбуждающего излучения (рис. 2 и 3), регистрируемого детектором D3 в небольшом телесном угле, задаваемом диафрагмой D (рис. 1). При распаде скалярных бифотонов на два „запутанных“ фотона, наоборот, наблюдается возрастание интенсивности направленного излучения, регистрируемого детектором D3 (рис. 2 и 3). Возрастание коэффициентов нелинейной рефракции следует ожидать при понижении порога ВКР, реализуемого в конденсированных диэлектрических средах при приближении к условиям резонансного возрастания эффективного сечения спонтанного комбинационного рассеяния, а также при использовании микрорезонаторного усиления в фотонных кристаллах и при реализации нелинейно-оптических резонансов Ми.

\section{Финансирование работы}

Работа выполнена за счет средств Российского научного фонда, проект № 19-12-00242.

\section{Конфликт интересов}

Авторы заявляют, что у них нет конфликта интересов.

\section{Список литературы}

[1] Zverev P.G., Basiev T.T., Osiko V.V., Kulkov A.M., Voitsekhovskii V.N., Yakobson V.E. // Opt. Mater. 1999. V. 11. P. 315.

[2] Zverev P.G., Basiev T.T., Prokhorov A.M. // Optical Materials. 1999. V. 11. P. 335.

[3] Orlovich V.A., Apanasevich P.A., Batishche S.A., Belyi V.N., Bui A.A., Grabchikov A.S., Kazak N.S., Kachinskii A.V. // J. Optical Technology. 2000. V. 67. P. 984.

[4] Basiev T.T., Osiko V.V., Prokhorov A.M., Dianov E.M. // Topics in Appl. Phys. 2003. V. 89. P. 459.

[5] Apanasevich P.A., Batishche S.A., Grabchikov A.S., Kuz'muk A.A., Lisinetskii V.A., Orlovich V.A., Tatur G.A., Chulkov R.V. // J. Appl. Spectrosc. 2006. V. 73. P. 371.

[6] Lisinetskii V.A., Grabtchikov A.S., Khodasevich I.A., Eichler H.J., Orlovich V.A. // Opt. Commun. 2007. V. 272. P. 509.

[7] Горелик В.С., Козулин Е.А. // Квант. электрон. 1994. Т. 2. P. 499; Gorelik V.S., Kozulin E.A. // Quant. Electron. 1994. V. 24. P. 462.

[8] Gorelik V.S., Agaltsov A.M., Zlobina L.I. // J. Mol. Structures. V. 266. 1992. P. 121.

[9] Buganov O.V., Grabtchikov A.S., Malakhov Y.I., Popov Y.M., Orlovich V.A., Tikhomirov S.A. // Laser Phys. Lett. 2012. V. 9. P. 786.

[10] Rumi M., Perry J.W. // Advances in Optics and Photonics. 2010. V. 2. P. 451.

[11] Amudha M., Madhavan J., Praveen Kumar. // J. Optics. 2017. V. 46. P. 382. 
[12] Sheik-Bahae M., Said A.A., Wei T.-H., Hagan D.J., Stryland E.W.V. // IEEE J. Quant. Electron. 1990. V. 26. P. 760 .

[13] Vodchits A.I., Kozich V.P., Orlovich V.A., Apanasevich P.A. // Opt. Commun. 2006. V. 263. P. 304.

[14] Vodchits A.I., Orlovich V.A., Apanasevich P.A., Basiev T.T., Zverev P.G. // Opt. Mater. 2007. V. 29. P. 1616.

[15] Gorelik V.S. // Acta Phys. Hung. 2006. V.A. P. 2637.

[16] Gorelik V.S. // J. Russ. Laser Research. 2006. V. 27. P. 400.

[17] Gorelik V.S. // Laser Phys. 2008. V. 18. P. 1479. 